\title{
A numerical study of the performance of alternative weighted ENO methods based on various numerical fluxes for conservation law*
}

\author{
Hongxia Liu ${ }^{\dagger}$
}

\begin{abstract}
In this paper, we systematically investigate the performance of the weighted essential non-oscillatory (WENO) methods based on various numerical fluxes for the nonlinear hyperbolic conservation law. Our objective is enhancing the performance for the conservation laws through picking out the suitable numerical fluxes. We focus our attention entirely on the comparison of eight numerical fluxes with the fifth-order accurate finite difference WENO methods and third-order accurate TVD Runge-Kutta time discretization for hyperbolic conservation laws. In addition, we give their implementation based on a new form framework of flux which we used in [9] and was proposed by Shu and Osher in [16]. The detailed numerical study is mainly implemented for the one dimensional system case, including the discussion of the CPU cost, accuracy, non-oscillatory property, and resolution of discontinuities. Numerical tests are also performed for two dimensional systems.
\end{abstract}

Key words: finite difference WENO scheme; numerical flux; Riemann solver; high-order accurate; hyperbolic conservation law

\section{Introduction}

We know that one of the important components of the WENO schemes $[8,6,14]$ for the conservation law is the numerical flux whose performance sometimes can directly affect the numerical result (of pros and cons). In this paper, we study an alternative flux formulation based on the finite difference WENO methods to solve the nonlinear hyperbolic conservation law

$$
\left\{\begin{array}{l}
u_{t}+\nabla \cdot f(u)=0, \\
u(x, 0)=u_{0}(x) .
\end{array}\right.
$$

* Research was supported by the National Natural Science Foundation of China and the School Young Foundation of Taiyuan University of Technology(2015QN060).

$\dagger$ College Of Mathematics, Taiyuan University of Technology, Taiyuan, Shanxi 030024, P.R. China. Email: hx_ryu@163.com 
The aim is to investigate the performance of different numerical fluxes based on the WENO methods, and to obtain better performance for the hyperbolic conservation laws by choosing suitable numerical fluxes. Moreover, in most of the WENO methods in the literature, the Lax-Friedrichs (LF) numerical flux was used due to its simplicity and easy implementation. Although LF flux is one of the simplest and most widely used for the high order finite difference/volume WENO methods, the numerical viscosity of the LF flux is also the largest among many other monotone fluxes. Thus we can not get a satisfactory numerical results, since the numerical viscosity may smooth many useful messages with the time development. There are many other numerical fluxes based on either exact or approximate Riemann solvers in the literature $[12,11,19,3,4,10,21,5]$, which could also be used in the context of the WENO methods.

We now give a brief survey of WENO schemes. WENO schemes are high order schemes for approximating hyperbolic conservation laws and other convection dominated partial differential equations. They can produce sharp, non-oscillatory discontinuity transitions and high order accurate resolutions for the smooth part of the solution. The first WENO scheme was introduced in 1994 by Liu, Osher, and Chan in their pioneering paper [8], in which a third order accurate finite volume WENO scheme in one space dimension was constructed. In [6], a general framework is provided to design arbitrary high order accurate finite difference WENO schemes, which are more efficient for multidimensional calculations. Very high order finite difference WENO schemes (seventh to eleventh order) are documented in [1].

However, for a finite difference WENO scheme using the flux splitting method to compute its numerical fluxes generally [14], it is difficult to implement the procedure for most two point monotone numerical fluxes because we do have not splitting each of them to satisfy the upwinding performance for the stability of the scheme. In [7], Jiang, Shu and Zhang designed a finite difference WENO scheme with Lax-Wendroff time discretization, avoiding the above mentioned difficulty.

In this work, we would like to explore the alternative formulation for constructing numerical fluxes in high order conservative finite difference schemes which involves interpolations directly on the point values of the solution $u_{i}$ rather than on the flux values. This alternative formulation, even though less clean and more computationally expensive, overcomes the above disadvantages, while enabling the use of monotone fluxes and interpolation directly on the points value. We take a numerical flux as being composed of two terms, a low and a high order flux. This approach has been proposed in reference [16] and used recently in reference [7]. As a low order flux we choose same typically used monotone numerical flux and add high order flux by Taylor expansion.

As summarized in [7], one of the advantages of this form flux is that arbitrary monotone 
fluxes can be used in this framework, while the traditional practice of constructing flux functions can be applied only to smooth flux splitting. Therefore, we can obtain an alternative formulations of conservation finite difference WENO schemes, where instead of a low order numerical flux $\hat{f}_{i+\frac{1}{2}}^{L}$ in $(2.5)$ we use eight different numerical fluxes.

The fluxes that are consider in this work are, the Godunov flux [4], the Engquist-Osher (EO) flux [3] (for the scalar case, its extension to systems referred to as the Osher-Solomon flux), the Harten, Lax and van Leer(HLL) flux [5], and a modification of the HLL flux, called HLLC flux. These fluxes are based on the approximation Riemann solver, are two point, first-order monotone fluxes; the others we study are essentially two point fluxes, they satisfy

the essential two point property: $\hat{f}\left(u^{l}, u, u, u^{r}\right)=f(u)$ for any $u^{l}$ and $u^{r}$, which can be viewed as the correction of a low order flux. A comparison is made between the different schemes based on the WENO scheme to approximate the hyperbolic conservation laws mainly for the one dimensional system case. The approximate Riemann solver of LF, HLL and HLLC are used in two dimensional systems. We review and describe the details of the numerical fluxes under consideration in section 2, and present extensive numerical experiments and compare their performance of these considered fluxes in section 3. Concluding remarks are given in section 4 .

\section{Review and implementation of the numerical fluxes for the WENO methods}

In this section we review the WENO method for spatial discretization of the conservation laws and the numerical fluxes under consideration and nonlinear stable Runge-Kutta methods for time. We start with the description of the WENO method in one dimensional scalar case.

Consider the one dimensional hyperbolic conservation law given by

$$
u_{t}+f(u)_{x}=0
$$

By taking the uniform mesh $x_{i+\frac{1}{2}}=x_{i}+\frac{\Delta x}{2}, x_{i}=\frac{1}{2}\left(x_{i-\frac{1}{2}}+x_{i+\frac{1}{2}}\right), i=1, \cdots, N$, where $\Delta x=\frac{1}{N}$, denoting the cell $I_{i}=\left[x_{i-\frac{1}{2}}, x_{i+\frac{1}{2}}\right]$, a semidiscrete conservative finite difference scheme for solving (2.1) has the form

$$
\frac{d u_{i}(t)}{d t}=-\frac{1}{\Delta x}\left(\hat{f}_{i+1 / 2}-\hat{f}_{i-1 / 2}\right)
$$

where $u_{i}(t)$ is the numerical approximation to the nodal value $u\left(x_{i}, t\right)$. The numerical flux

$$
\hat{f}_{i+\frac{1}{2}}=\hat{f}\left(u_{i-r}, \ldots, u_{i+s}\right)
$$


is designed by

$$
\frac{1}{\Delta x}\left(\hat{f}_{i+1 / 2}-\hat{f}_{i-1 / 2}\right)=\left.f(u(x))_{x}\right|_{x_{i}}+O\left(\Delta x^{k}\right)
$$

for a k-order scheme. In order to obtain higher order schemes $(k \geq 3)$, the finite difference WENO schemes customarily introduce an integrand $h(x)$, then use the fundamental theorem of calculus, to obtain a high-order accurate numerical flux $\hat{f}_{i+1 / 2}$. This approach uses Lemma 2.1 in [14], for details see also [6, 15]. However, a disadvantage of using the Lemma 2.1 is that we need to choose a suitable numerical flux which can split incorporated upwinding of the scheme for achieving the nonlinear stability.

In view of these considerations, we take another new form of numerical flux, originally designed in [16], to be used again recently in [7]. Within this modality we choose some typically used monotone numerical flux as a low order flux $\hat{f}_{i+\frac{1}{2}}^{L}$ and add a high order flux $\hat{f}_{i+\frac{1}{2}}^{H}$ by Taylor expansion. Thus we obtain a high resolution numerical flux $\hat{f}_{i+\frac{1}{2}}$.

We can view the high order flux as consisting of the low order flux plus a correction:

$$
\hat{f}_{i+\frac{1}{2}}=\hat{f}_{i+\frac{1}{2}}^{L}+\hat{f}_{i+\frac{1}{2}}^{H}
$$

where the low order flux $\hat{f}_{i+\frac{1}{2}}^{L}$ and the high order flux $\hat{f}_{i+\frac{1}{2}}^{H}$ are defined by

$$
\begin{aligned}
& \hat{f}_{i+\frac{1}{2}}^{L}=h\left(u_{i+\frac{1}{2}}^{-}, u_{i+\frac{1}{2}}^{+}\right) \\
& \hat{f}_{i+\frac{1}{2}}^{H}=\sum_{l=1}^{(m-1) / 2} a_{2 l} \Delta x^{2 l}\left(\frac{\partial^{2 l} f}{\partial x^{2 l}}\right)_{i+\frac{1}{2}}+O\left(\Delta x^{m}\right) .
\end{aligned}
$$

Denoting the $f_{i}^{n}$ as the approximation of the point value $f\left(u\left(x_{i}, t^{n}\right)\right)$ and $f^{(l)}$ as the $l$-th order spatial derivative of $f, f^{(l)}=\frac{\partial^{l} f}{\partial x^{l}}$. According to reference [16], there exist constants $a_{2}, a_{4}, \ldots$ such that the numerical flux (2.5) guarantees $k=m$-th order accuracy in (2.4). The coefficients $a_{2 l}$ in (2.7) can be obtained by Taylor expansion the left hand side of (2.4).

We can rewrite the conservation law (2.1) by a semi-discrete form of (2.2) and adopt the flux form of (2.5). The time evolution is treated by the third order nonlinear stable Runge-Kutta schemes [16].

For $k=5$ in Eq. (2.4), the numerical flux function in Eq. (2.2) can be computed using the fifth-order WENO schemes [7]. The low resolution term of the numerical flux $\hat{f}_{i+\frac{1}{2}}^{L}$ in (2.6) is given by the eight different numerical fluxes listed in section 2.3 , where the values $u_{i+\frac{1}{2}}^{ \pm}$in $h\left(u_{i+\frac{1}{2}}^{-}, u_{i+\frac{1}{2}}^{+}\right)$are obtained by the classical fifth-order finite difference WENO schemes. For completeness, the process of the WENO reconstruction is given in the following subsection.

Remark: To the low order numerical flux $\hat{f}_{i+\frac{1}{2}}^{L}$ in (2.5), we can use any monotone flux, this is the one of key benefits of this alternative flux formulation. Meanwhile, we also demonstrate some other results such as the comparison of the alternative WENO method (in 
which use LF flux) and standard WENO-LF discretization to some benchmark examples, to illustrate the alternative performance of our schemes.

\subsection{Description of the low resolution terms: WENO reconstruc- tion.}

Given the nodal values $u_{i}=u\left(x_{i}\right)$ of a piecewise smooth function $u(x)$, we would like to find a high-order accurate approximation of $u(x)$ at the half points $x_{i+\frac{1}{2}}$. The formulae of the WENO scheme can be expressed as

$$
u_{i+\frac{1}{2}}^{-}=\sum_{r=0}^{k-1} \omega_{r} u_{i+\frac{1}{2}}^{(r)}
$$

where $u_{i+\frac{1}{2}}^{(r)}$ is obtained by a $(k-1)$-th order polynomial reconstruction of $u\left(x_{i+\frac{1}{2}}\right)$ (denoted by $\left.p_{r}(x), p_{r}\left(x_{j}\right)=u_{j}\right)$ on $r$-th set of candidate stencils $S_{r} . S_{r}$ is the small stencil, $S_{r}=$ $\left\{x_{i-s}, x_{i-s+1}, \ldots, x_{i+r}\right\}$ for $r=0,1, \ldots, k-1$ with $r+s=k-1$, which cover all possible stencils including the grid point that one point upwind to $x_{i+\frac{1}{2}}$. For each $r, \omega_{r}$ is the nonlinear weight that satisfies

$$
\sum_{r=0}^{k-1} \omega_{r}=1, \quad \omega_{r} \geq 0 .
$$

Following the original WENO recipe [6], $\omega_{r}$ is given by

$$
\omega_{r}=\frac{\bar{\omega}_{r}}{\sum_{s=0}^{k-1} \bar{\omega}_{r}}, \quad r=0,1, \ldots, k-1
$$

with

$$
\bar{\omega}_{r}=\frac{\gamma_{r}}{\left(\varepsilon+\beta_{r}\right)^{2}},
$$

where $\gamma_{r}$ is a linear weight which guarantees that the overall scheme (2.8) is $k$-order accurate. And $\beta_{r}$ is the smoothness indicator, defined by

$$
\beta_{r}=\sum_{l=1}^{k-1} \int_{I_{i}} \Delta x^{2 l-1}\left(\frac{\partial^{l} p_{r}(x)}{\partial^{l} x}\right)^{2} d x .
$$

We take $\varepsilon=10^{-6}$ (a small positive number) to avoid division by zero.

Especially, for the case $k=3$, we have

$$
\gamma_{0}=\frac{1}{16}, \quad \gamma_{1}=\frac{5}{8}, \quad \gamma_{2}=\frac{5}{16}
$$


And $u_{i+\frac{1}{2}}^{-}, \beta_{r}(r=0,1,2)$ can be computed by

$$
\begin{aligned}
& u_{i+\frac{1}{2}}^{(0)}=\frac{3}{8} u_{i-2}-\frac{5}{4} u_{i-1}+\frac{15}{8} u_{i}, \\
& u_{i+\frac{1}{2}}^{(1)}=-\frac{1}{8} u_{i-1}+\frac{3}{4} u_{i}+\frac{3}{8} u_{i+1}, \\
& u_{i+\frac{1}{2}}^{(2)}=\frac{3}{8} u_{i}+\frac{3}{4} u_{i+1}-\frac{1}{8} u_{i+2}
\end{aligned}
$$

and

$$
\begin{aligned}
& \beta_{0}=\frac{13}{12}\left(u_{i-2}-2 u_{i-1}+u_{i}\right)^{2}+\frac{1}{4}\left(u_{i-2}-4 u_{i-1}+3 u_{i}\right)^{2} \\
& \beta_{1}=\frac{13}{12}\left(u_{i-1}-2 u_{i}+u_{i+1}\right)^{2}+\frac{1}{4}\left(u_{i-1}-u_{i+1}\right)^{2} \\
& \beta_{2}=\frac{13}{12}\left(u_{i}-2 u_{i+1}+u_{i+2}\right)^{2}+\frac{1}{4}\left(3 u_{i}-4 u_{i+1}+u_{i+2}\right)^{2} .
\end{aligned}
$$

The reconstruction to $u_{i+\frac{1}{2}}^{+}$is mirror symmetric to that for $u_{i+\frac{1}{2}}^{-}$, and we have

$$
\gamma_{0}=\frac{5}{16}, \quad \gamma_{1}=\frac{5}{8}, \quad \gamma_{2}=\frac{1}{16}
$$

The formulae for $u_{i+\frac{1}{2}}^{+}, \beta_{r}(r=0,1,2)$ become

$$
\begin{aligned}
u_{i+\frac{1}{2}}^{(0)} & =-\frac{1}{8} u_{i-1}+\frac{3}{4} u_{i}+\frac{3}{8} u_{i+1}, \\
u_{i+\frac{1}{2}}^{(1)} & =\frac{3}{8} u_{i}+\frac{3}{4} u_{i+1}-\frac{1}{8} u_{i+2}, \\
u_{i+\frac{1}{2}}^{(2)} & =\frac{15}{8} u_{i+1}-\frac{5}{4} u_{i+2}+\frac{3}{8} u_{i+3}
\end{aligned}
$$

and

$$
\begin{aligned}
& \beta_{0}=\frac{13}{12}\left(u_{i-1}-2 u_{i}+u_{i+1}\right)^{2}+\frac{1}{4}\left(u_{i-1}-4 u_{i}+3 u_{i+1}\right)^{2}, \\
& \beta_{1}=\frac{13}{12}\left(u_{i}-2 u_{i+1}+u_{i+2}\right)^{2}+\frac{1}{4}\left(u_{i}-u_{i+2}\right)^{2}, \\
& \beta_{2}=\frac{13}{12}\left(u_{i+1}-2 u_{i+2}+u_{i+3}\right)^{2}+\frac{1}{4}\left(3 u_{i+1}-4 u_{i+2}+u_{i+3}\right)^{2} .
\end{aligned}
$$

\subsection{Description of the high resolution terms.}

In order to guarantee $k=5$ th order accuracy in (2.4), we can use the first two terms in (2.7) given as

$$
\hat{f}_{i+\frac{1}{2}}^{H}=-\frac{1}{24} \Delta x^{2}\left(\frac{\partial^{2} f}{\partial x^{2}}\right)_{i+\frac{1}{2}}+\frac{7}{5760} \Delta x^{4}\left(\frac{\partial^{4} f}{\partial x^{4}}\right)_{i+\frac{1}{2}} .
$$

Noting that the high resolution term $\hat{f}_{i+\frac{1}{2}}^{H}$ of the numerical flux contains at least $\Delta x^{2}$ in its coefficients, therefore, the lower approximations are satisfactory as their contribution to 
spurious oscillations is weaker. Hence, the high resolution terms $\hat{f}_{i+\frac{1}{2}}^{H}$ are approximated by simple central difference with a suitable order of accuracy considering the one-point upwindbiased quality (as shown in [13]) and more costly WENO interpolation was not used.

Considering the upwinding quality, the spatial variable $f$ is decomposed into two parts

$$
f=f^{+}+f^{-}
$$

with

$$
\left(f^{+}\right)^{\prime} \geq 0,\left(f^{-}\right)^{\prime} \leq 0
$$

where

$$
f^{+}=\frac{1}{2}(f(u)+\alpha u), f^{-}=\frac{1}{2}(f(u)-\alpha u),
$$

and $\alpha \geq \max \left|f^{\prime}(u)\right|$ is a constant. Then clearly

$$
f^{+}\left(u_{i}\right)+f^{-}\left(u_{i}\right)=f\left(u_{i}\right) .
$$

We interpolate polynomials to approximate the high resolution terms based on the point values of $f^{+}\left(u_{i}\right)$ and $f^{-}\left(u_{i}\right)$ with the simple central difference without the WENO procedure.

We construct the numerical flux $\hat{f}_{i+\frac{1}{2}}^{L}$ with $(2 r-1)$-th order accuracy as described in section 2.1. The WENO interpolation $u_{i+\frac{1}{2}}^{ \pm}$are obtained based on the points $\left\{x_{i-r+1}, \ldots, x_{i+r}\right\}$. For the numerical fluxes $\hat{f}_{i+\frac{1}{2}}^{H}$, the derivatives $\frac{\partial^{k} f}{\partial x^{k}}$ at the half points $x_{i+\frac{1}{2}}$, we use $(2 r-1-k)$ th order finite difference approximations based on the same points $\left\{x_{i-r+1}, \ldots, x_{i+r}\right\}$. For $r=3$, we can obtain the fifth accuracy order approximation to the high order derivative terms. The numerical flux values corresponding to the two terms in (2.11) at $x_{i+\frac{1}{2}}$ are obtained from the standard polynomial interpolation based on the same points $\left\{x_{i-2}, \ldots, x_{i+3}\right\}$.

The approximation of the second derivative term is

$$
\hat{f}_{i+\frac{1}{2}}^{(1)}=-\left.\frac{\Delta x^{2}}{24} \frac{\partial^{2} f}{\partial x^{2}}\right|_{x_{i+\frac{1}{2}}}=\frac{1}{24}\left(\hat{f}_{i+\frac{1}{2}}^{+}+\hat{f}_{i+\frac{1}{2}}^{-}\right)
$$

where

$$
\begin{aligned}
& \hat{f}_{i+\frac{1}{2}}^{+}=-\frac{5}{24} f_{i-2}^{+}+\frac{4}{3} f_{i-1}^{+}-\frac{7}{4} f_{i}^{+}+\frac{1}{3} f_{i+1}^{+}+\frac{7}{24} f_{i+2}^{+}, \\
& \hat{f}_{i+\frac{1}{2}}^{-}=\frac{7}{24} f_{i-1}^{-}+\frac{1}{3} f_{i}^{-}-\frac{7}{4} f_{i+1}^{-}+\frac{4}{3} f_{i+2}^{-}-\frac{5}{24} f_{i+3}^{-} .
\end{aligned}
$$

For the approximation of the fourth derivative, we have

$$
\hat{f}_{i+\frac{1}{2}}^{(2)}=\left.\frac{7}{5760} \Delta x^{4} \frac{\partial^{4} f}{\partial x^{4}}\right|_{x_{i+\frac{1}{2}}}=\frac{7}{5760}\left(\hat{f}_{i+\frac{1}{2}}^{+}+\hat{f}_{i+\frac{1}{2}}^{-}\right)
$$


where

$$
\begin{aligned}
& \hat{f}_{i+\frac{1}{2}}^{+}=f_{i-2}^{+}-4 f_{i-1}^{+}+6 f_{i}^{+}-4 f_{i+1}^{+}+f_{i+2}^{+}, \\
& \hat{f}_{i+\frac{1}{2}}^{-}=f_{i-1}^{-}-4 f_{i}^{-}+6 f_{i+1}^{-}-4 f_{i+2}^{-}+f_{i+3}^{-} .
\end{aligned}
$$

Finally, the high resolution numerical flux is formed by

$$
\hat{f}_{i+\frac{1}{2}}^{H}=\hat{f}_{i+\frac{1}{2}}^{(1)}+\hat{f}_{i+\frac{1}{2}}^{(2)}
$$

\subsection{Description of fluxes for the high resolution schemes}

We now review the two point or essentially two point numerical fluxes under consideration. Numerical tests to compare their performance for our scheme will be given in next section. For the case of one dimensional system, we will consider Euler equations of compressible gas dynamics, namely (2.1) with

$$
u=(\rho, \rho v, E)^{T}, \quad f(u)=\left(\rho v, \rho v^{2}+p, v(E+p)\right)
$$

where $\rho$ is the density, $v$ is the velocity, $E$ is the total energy, $p$ is the pressure, which is related to the total energy by $E=\frac{p}{\gamma-1}+\frac{1}{2} \rho v^{2}$ with $\gamma=1.4$ for air. We will also use the sound velocity $c=\sqrt{\frac{\gamma p}{\rho}}$ in the definition of some of the numerical fluxes.

1. The Lax-Friedrichs (LF) flux and the local LF (LLF) flux.

Although the LF flux is one of the simplest and most widely used building blocks for the high order finite difference/volume methods such as WENO, ENO or DG schemes, the numerical viscosity of the LF flux is also the largest among many other monotone fluxes. Often we can not get satisfactory numerical results since the numerical viscosity smooth many useful messages with the time development.

The LF or the LLF fluxes are defined by:

$$
\hat{f}^{L F}\left(u^{-}, u^{+}\right)=\frac{1}{2}\left[\left(f\left(u^{-}\right)+f\left(u^{+}\right)\right)-\alpha\left(u^{+}-u^{-}\right)\right],
$$

where for the LF flux, $\alpha$ is taken as an upper bound over the whole line for $\left|f^{\prime}(u)\right|$ in the scalar case, or for the absolute value of eigenvalues of the Jacobian for the system case, and $\alpha$ is taken as an upper bound between $u^{-}$and $u^{+}$for the LLF flux .

2. The Godunov flux.

The Godunov flux $[4,19]$ is based on the exact Riemann solver, which has the smallest numerical viscosity among all monotone fluxes for the scalar case but could be very 
costly to evaluate in the system case, as it often lacks explicit formulas and relies on iterative procedures for its evaluation. The Godunov flux is defined as

$$
\hat{f}^{G}\left(u^{-}, u^{+}\right)=f(u(0)),
$$

where $u(0)$ is the solution of the local Riemann problem at $x / t=0$ (the solution of the local Riemann problem is a function of the single variable $x / t$ only due to self-similarity), i.e. the exact solution to the conservation law (2.1) with the initial condition:

$$
u(x, 0)= \begin{cases}u^{-} & \text {for } \quad x \leq 0, \\ u^{+} & \text {for } \quad x>0 .\end{cases}
$$

For the scalar case, the Godunov flux can be expressed in a closed form as

$$
\hat{f}^{G}\left(u^{-}, u^{+}\right)=\left\{\begin{array}{lll}
\min _{u^{-} \leq u \leq u^{+}} f(u) & \text { if } u^{-} \leq u^{+}, \\
\max _{u^{+} \leq u \leq u^{-}} f(u) & \text { if } \quad u^{-}>u^{+} .
\end{array}\right.
$$

However, for most nonlinear systems, the Godunov flux cannot be expressed in a closed form. Its evaluation would in general require an iterative procedure. We refer to [19] and the references therein for more details about the exact Riemann solver for systems in applications.

3. The Engquist-Osher (EO) flux and the Osher-Solomon flux [3, 10].

The Engquist-Osher (EO) flux for the scalar case and its extension to systems (often referred to as the Osher-Solomon flux ) are smoother than the Godunov flux with an almost as small numerical viscosity, and have the advantage of explicit formulas for the scalar case and for some well known physical systems, such as the Euler equations of compressible gas dynamics. Since the existence of the explicit formulas, the evaluation of the EO flux is less costly than the Godunov flux.

For the scalar case the EO flux is given by:

$$
\hat{f}^{E O}\left(u^{-}, u^{+}\right)=\frac{1}{2}\left(f\left(u^{-}\right)+f\left(u^{+}\right)-\int_{u^{-}}^{u^{+}}\left|f^{\prime}(u)\right| d u\right) .
$$

In the case of one dimensional system, the explicit formulas for the Osher-Solomon flux for the Euler equations (2.1) are given as follows: first we compute intermediate variables based on $u^{ \pm}$:

$$
\begin{aligned}
& \rho_{1}=\rho^{+}\left(\frac{(\gamma-1)\left(v^{+}-v^{-}\right) / 2+c^{+}+c^{-}}{c^{+}\left(1+\left(p^{-} / p^{+}\right)^{1 / 2 \gamma}\left(\rho^{-} / \rho^{+}\right)^{-1 / 2}\right)}\right)^{2 /(\gamma-1)}, \\
& \rho_{2}=\rho^{-}\left(\frac{(\gamma-1)\left(v^{+}-v^{-}\right) / 2+c^{+}+c^{-}}{c^{-}\left(1+\left(p^{+} / p^{-}\right)^{1 / 2 \gamma}\left(\rho^{+} / \rho^{-}\right)^{-1 / 2}\right)}\right)^{2 /(\gamma-1)},
\end{aligned}
$$




$$
\begin{aligned}
& p_{1}=p_{2}=p^{-}\left(\frac{\rho^{-}}{\rho_{2}}\right)^{-\gamma}, \quad v_{1}=v_{2}=v^{-}-\frac{2}{\gamma-1}\left(c^{-}-\sqrt{\frac{\gamma p_{2}}{\rho_{2}}}\right) \\
& \bar{\rho}_{1}=\rho^{+}\left(\frac{(\gamma-1) v^{+}+2 c^{+}}{(\gamma+1) c^{+}}\right)^{2 /(\gamma-1)}, \quad \bar{\rho}_{2}=\rho^{-}\left(\frac{-(\gamma-1) v^{-}+2 c^{-}}{(\gamma+1) c^{-}}\right)^{2 /(\gamma-1)}, \\
& \bar{p}_{1}=p^{+}\left(\bar{\rho}_{1} / \rho^{+}\right)^{\gamma}, \quad \bar{p}_{2}=p^{-}\left(\bar{\rho}_{2} / \rho^{-}\right)^{\gamma}, \\
& \bar{v}_{1}=v^{+}+\frac{2}{\gamma-1}\left(c^{+}-\sqrt{\gamma \bar{p}_{1} / \bar{\rho}_{1}}\right), \quad \bar{v}_{2}=v^{-}-\frac{2}{\gamma-1}\left(c^{-}-\sqrt{\gamma \bar{p}_{2} / \bar{\rho}_{2}}\right) .
\end{aligned}
$$

The Osher-Solomon flux is then still given by (2.18), with the integral computed as a sum of three parts based on three characteristic fields:

$$
\int_{u^{-}}^{u^{+}}\left|f^{\prime}(u)\right| d u=\int_{\Gamma_{1}}+\int_{\Gamma_{2}}+\int_{\Gamma_{3}}\left(f^{\prime}(u)^{+}-f^{\prime}(u)^{-}\right) d u,
$$

where:

$$
\begin{aligned}
& \int_{\Gamma_{1}} f^{\prime}(u)^{+} d u=f(u)\left|\begin{array}{lll}
u^{+} & \text {if } & \rho^{+}<\bar{\rho}_{1}, \\
\bar{u}_{1} & \text { if } & \rho^{+} \geq \bar{\rho}_{1}, \\
& &
\end{array} \quad, \quad \int_{\Gamma_{1}} f^{\prime}(u)^{-} d u=f(u)\right| \begin{array}{lll}
u^{+} & \text {if } & \rho^{+} \geq \bar{\rho}_{1}, \\
\bar{u}_{1} & \text { if } & \rho^{+}<\bar{\rho}_{1}, \\
& & \\
u_{1} & \text { if } & \rho_{1}<\bar{\rho}_{1}, \\
\bar{u}_{1} & \text { if } & \rho_{1} \geq \bar{\rho}_{1} .
\end{array} \quad \begin{array}{lll}
u_{1} & \text { if } & \rho_{1} \geq \bar{\rho}_{1}, \\
\bar{u}_{1} & \text { if } & \rho_{1}<\bar{\rho}_{1} .
\end{array}, \\
& \int_{\Gamma_{2}}\left(f^{\prime}(u)^{+}-f^{\prime}(u)^{-}\right) d u=\left(\rho_{1}-\rho_{2}\right)\left|v_{1}\right|\left(\begin{array}{c}
1 \\
v_{1} \\
v_{1}^{2} / 2
\end{array}\right) .
\end{aligned}
$$

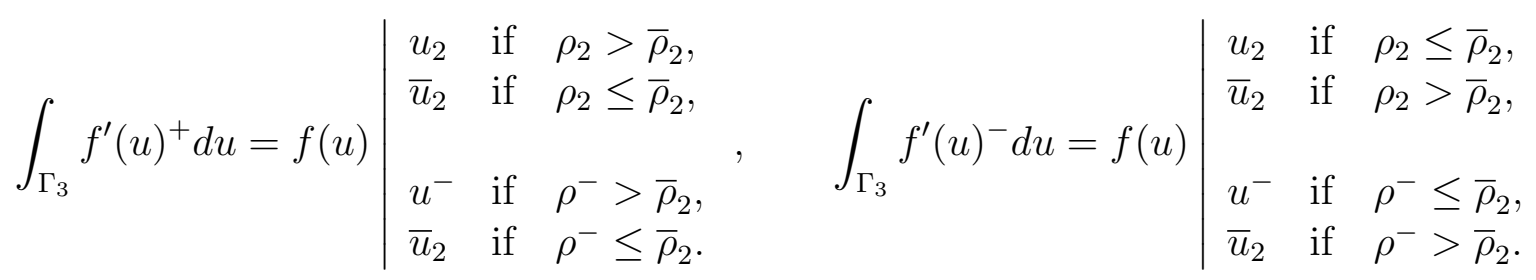

For the multidimensional formula, the extension of the Osher scheme problem is straightforward (see [19] for more details).

4. The Harten-Lax-van Leer (HLL) flux [5, 19].

For the purpose of computing a Godunov flux, Harten, Lax and van Leer [5] presented a novel approach (HLL flux) for solving the Riemann problem approximately. The central idea is to assume, for the solution, a wave configuration that consists of two waves separating three constant states. The HLL flux for the Euler equations (2.15) (one dimensional case) is defined by:

$$
\hat{f}^{H L L}\left(u^{-}, u^{+}\right)= \begin{cases}f\left(u^{-}\right), & \text {if } 0 \leq s^{-} \\ \frac{s^{+} f\left(u^{-}\right)-s^{-} f\left(u^{+}\right)+s^{-} s^{+}\left(u^{+}-u^{-}\right)}{s^{+}-s^{-}}, & \text {if } s^{-} \leq 0 \leq s^{+} \\ f\left(u^{+}\right), & \text {if } s^{+} \leq 0\end{cases}
$$


where the lower and upper bounds of the wave velocity, $s^{-}$and $s^{+}$, must be estimated. We use the pressure-velocity estimates

$$
s^{-}=v^{-}-c^{-} q^{-}, \quad s^{*}=v^{*}, \quad s^{+}=v^{+}+c^{+} q^{+}
$$

where, for $K= \pm$,

$$
q^{K}= \begin{cases}1, & \text { if } \quad p^{*} \leq p^{K} \\ \left(1+\frac{\gamma+1}{2 \gamma}\left(p^{*} / p^{K}-1\right)\right)^{1 / 2}, & \text { if } \quad p^{*}>p^{K}\end{cases}
$$

with

$$
p^{*}=\frac{1}{2}\left(p^{-}+p^{+}\right)-\frac{1}{2}\left(v^{+}-v^{-}\right) \bar{\rho} \bar{c}, \quad v^{*}=\frac{1}{2}\left(v^{-}+v^{+}\right)-\frac{p^{+}-p^{-}}{2 \bar{\rho} \bar{c}},
$$

and

$$
\bar{\rho}=\frac{1}{2}\left(\rho^{-}+\rho+\right), \quad \bar{c}=\frac{1}{2}\left(c^{-}+c^{+}\right) .
$$

However this flux has a serious flaw of diffusing contact surfaces. This is mainly because the HLL scheme is an assumption of a two wave configuration, therefore neglecting the contacts discontinuity. To remedy this problem a version of intermediate waves in the HLL scheme was given in [21], named HLLC flux.

5. The HLLC flux - a modification of the HLL flux [21, 19].

The HLLC flux is a modification of the HLL flux, whereby the missing contact and shear waves are restored. The HLLC flux for the Euler equations (2.15) is given by:

$$
\hat{f}^{H L L C}\left(u^{-}, u^{+}\right)= \begin{cases}f\left(u^{-}\right), & \text {if } 0 \leq s^{-}, \\ f\left(u^{-}\right)+s^{-}\left(u^{*-}-u^{-}\right), & \text {if } s^{-} \leq 0 \leq s^{*} \\ f\left(u^{+}\right)+s^{+}\left(u^{*+}-u^{+}\right), & \text {if } s^{*} \leq \leq \leq s^{+} \\ f\left(u^{+}\right), & \text {if } s^{+} \leq 0\end{cases}
$$

where, for $K= \pm$,

$$
u^{* K}=\rho^{K} \frac{s^{K}-v^{K}}{s^{K}-s^{*}}\left[\begin{array}{c}
1 \\
s^{*} \\
\frac{E^{K}}{\rho^{K}}+\left(s^{*}-v^{K}\right)\left[s^{*}+\frac{p^{K}}{\rho^{K}\left(s^{K}-v^{K}\right)}\right]
\end{array}\right]
$$

The definitions of $s^{-}, s^{*}$ and $s^{+}$are given in (2.21). For the multidimensional formula see e.g. $[19,2]$.

6. The first-order centered (FORCE) flux [19].

This flux is the average of the LF flux and the second order Richtmyer flux, hence its viscosity is smaller than that of the LF flux. The FORCE flux is defined by:

$$
\hat{f}^{F O R C E}\left(u^{-}, u^{+}\right)=\frac{1}{2}\left(\hat{f}^{L F}\left(u^{-}, u^{+}\right)+\hat{f}^{R}\left(u^{-}, u^{+}\right)\right)
$$


where $\hat{f}^{R}$ is the second order Richtmyer flux given by

$$
\hat{f}^{R}\left(u^{-}, u^{+}\right)=f\left(u^{*}\right), \quad u^{*}=\frac{1}{2}\left(u^{-}+u^{+}-\frac{\Delta t}{\Delta x}\left(f\left(u^{+}\right)-f\left(u^{-}\right)\right)\right) .
$$

7. A flux limiter centered (FLIC) flux [19].

The general flux limiter approach combines a low order monotone flux and a high order flux. The FLIC flux we use has the FORCE flux as the low order flux and the Richtmyer flux as the high order flux:

$$
\hat{f}^{F L I C}\left(u^{-}, u^{+}\right)=\hat{f}^{F O R C E}\left(u^{-}, u^{+}\right)+\phi_{i+1 / 2}\left[\hat{f}^{R}\left(u^{-}, u^{+}\right)-\hat{f}^{F O R C E}\left(u^{-}, u^{+}\right)\right] .
$$

where $\phi_{i+1 / 2}$ is a flux limiter. There are several possible choices for the flux limiter such as the superbee, van Leer and the minbee flux limiters. Following [19, 18], for the Euler equation we use the following procedure: we first define $q=E$ (total energy) and set

$$
r_{i+1 / 2}^{-}=\frac{\Delta q_{i-1 / 2}}{\Delta q_{i+1 / 2}}, \quad r_{i+1 / 2}^{+}=\frac{\Delta q_{i+3 / 2}}{\Delta q_{i+1 / 2}}
$$

where $\Delta q_{i-1 / 2}=\bar{q}_{i}-\bar{q}_{i-1}$, and $\bar{q}_{i}$ is the cell average of $q$ on the cell $I_{i}$. We then compute a single flux limiter

$$
\phi_{i+1 / 2}=\min \left(\phi\left(r_{i+1 / 2}^{-}\right), \phi\left(r_{i+1 / 2}^{+}\right)\right)
$$

and apply it to all components of the flux. In this paper we use the minbee limiter:

$$
\phi(r)= \begin{cases}0, & r \leq 0 \\ r, & 0 \leq r \leq 1 \\ 1, & r \geq 1\end{cases}
$$

Clearly, if $u^{-}=u^{+}=u$, then $\hat{f}^{F L I C}(u, u)=f(u)$. Hence even if the FLIC flux depends on more than the two points $u^{-}$and $u^{+}$through the limiter $\phi_{i+1 / 2}$ and we are abusing notations when we denote it by $\hat{f}^{F L I C}\left(u^{-}, u^{+}\right)$, it is indeed an essentially two point flux as defined before.

8. The multi-stage predictor-corrector (MUSTA) flux [20].

The MUSTA flux is a multi-stage predictor-corrector flux. Following [18] we use the FORCE flux as the predictor flux. The procedure to evaluate a $L$-stage MUSTA flux can be described as following: first we set $u_{0}^{-}=u^{-}$and $u_{0}^{+}=u^{+}$for the initial stage $l=0$, then we perform the following steps:

(a) Compute the FORCE flux $\hat{f}_{l}^{F O R C E}=\hat{f}^{F O R C E}\left(u_{l}^{-}, u_{l}^{+}\right)$on the data at the stage $l$. 
(b) If the desired number of total stages $L$ has been reached (that is $l=L$ ), then the computation of the MUSTA flux is complete and the final flux is given by $\hat{f}^{M U S T A}\left(u^{-}, u^{+}\right)=\hat{f}_{l}^{F O R C E}$. Otherwise, continue to compute the values for the next stage using:

$$
u_{l+1}^{-}=u_{l}^{-}-\frac{\Delta t}{\Delta x}\left(\hat{f}_{l}^{F O R C E}-f\left(u_{l}^{-}\right)\right), \quad u_{l+1}^{+}=u_{l}^{+}-\frac{\Delta t}{\Delta x}\left(f\left(u_{l}^{+}\right)-\hat{f}_{l}^{F O R C E}\right)
$$

and proceed back to step (a).

In this paper we use $L=2$ as suggested in [18].

\section{Numerical results}

In this section we perform some known example tests to compare the performance of the eight different fluxes outlined in the previous section. The detailed numerical study is mainly performed for the one-dimensional system case, addressing the issues of $\mathrm{CPU}$ cost, accuracy, non-oscillatory property, and resolution of discontinuities. Numerical tests are also performed for two-dimensional systems. We performed our study on a Compaq Digital personal computer, intel core i5 with $2.6 \mathrm{GHz}$ for one-dimensional cases and on the Lenovo DeepComp 1800 Cluster for two-dimensional cases. In our numerical tests, the CFL numerical is taken 0.8 for one-dimensional cases and 0.2 for two-dimensional cases. The comparison of performance of all different fluxes is given in the following subsections.

Example 3.1. We solve the nonlinear system of Euler equations (2.15), where the initial condition is set to $\rho(x, t)=1+0.2 \sin (\pi(x-t)), v(x, t)=1, p(x, t)=1$, with a periodic boundary condition. The domain of interest in the $x-t$ plane are points $(x, t)$ which we choose $-1<x<1$ and $t>0$. We compute the solution up to $t=2$. In Table 3.1, we give the CPU time comparison for this paper's scheme using different fluxes. The numerical errors and the orders of accuracy for the density $\rho$, and ratios of the numerical errors for comparison with the LF flux are shown in Table 3.2.

Analysing the results, we observe that WENO-LF costs relatively little CPU time while it has relatively more numerical errors. The WENO-MUSTA scheme costs more CPU time and has the largest numerical errors, while the WENO-HLL and WENO-HLLC scheme costs less time and have considerable numerical errors.

Specifically, regarding the CPU time, the WENO-Godunov scheme costs several times than that of WENO-LF scheme. The WENO-EO and WENO-MUSTA schemes need about twice the CPU time than the WENO-LF scheme. The WENO-HLL and WENO-FORCE schemes costs are a little higher than that of the WENO-LF scheme. The WENO-LLF, 
Table 3.1: CPU time (in seconds) for the WENO methods with different fluxes, for the accuracy test problem. Total CPU time for $N=10,20,40,80$ and 160 cells is recorded.

\begin{tabular}{|cc|cc|cc|cc|cc|}
\hline flux & time & flux & time & flux & time & flux & time & flux & time \\
\hline LF & 0.4531 & EO & 0.8853 & HLLC & 0.5781 & FLIC & 0.5469 & LLF & 0.5528 \\
G & 1.0125 & HLL & 0.5314 & FORCE & 0.4688 & MUSTA & 0.7656 & - & - \\
\hline
\end{tabular}

WENO-FLIC and WENO-HLLC schemes cost about $20 \%$ and $25 \%$ more than the WENOLF scheme. From the numerical errors aspect, the $L_{1}$ and $L_{\infty}$ errors of WENO-HLL and WENO-FORCE schemes for the same grids are about $110 \%-120 \%$ of that by the WENOLF scheme. All the other schemes have the errors of about $90 \%$ or more than the WENO-LF scheme.

In our analysis, both the CPU time comparison and the numerical error comparison depend on our specific implementation of these fluxes and also on the specific test example, but it affords the correct ball-park of the CPU costs based on the WENO method using these different fluxes. Specifically, the Godunov flux has an iteration procedure and may converge with different number of steps for different solutions, hence the CPU time difference.

Example 3.2. Lax problem. We solve the same one dimensional Euler equations with a Riemann initial condition for the Lax problem

$$
(\rho, v, p)= \begin{cases}(0.445,0.698,3.528) & \text { for } x \leq 0 \\ (0.5,0,0.571) & \text { for } x>0\end{cases}
$$

In these computations we take the computational domain in the interval $-5 \leq x \leq 5$. The results are shown in Fig. 3.1 at the output time $t=1.3$. In each panel we compare each of the eight numerical fluxes considered with the LF numerical flux and the exact solution.

The results of Fig. 3.1 are in many ways representative of the quality of each flux based on our scheme. From the results, we can see that the WENO-HLLC, the WENO-Godunov and the WENO-EO schemes are slightly better than the WENO-LF scheme. In Fig. 3.2, by comparing with standard WENO-LF discretization scheme, we can observe that a similar results is obtained by using our scheme.

Example 3.3. Shu-Osher problem. In this example we consider the initial data for Euler equation (2.1) consisting of shock interaction with entropy wave, namely

$$
(\rho, v, p)= \begin{cases}(3.857143,2.629369,10.333333) & \text { for } x \leq 0 \\ (1+\varepsilon \sin (5 x), 0,1) & \text { for } x>0 .\end{cases}
$$

Here, we take the $\varepsilon=0.2$ and the computational domain $-5 \leq x \leq 5$ with 300 points. The computed densities for the output time $t=1.8$ are shown in Fig. 3.3. The solid line in this figure is obtained by computing the same problem using the WENO scheme with $N=2000$ grid points, which is considered to be the "exact" solution although the real exact solution 
Table 3.2: Euler equation, $\rho(x, 0)=1+0.2 \sin (\pi x), v(x, 0)=1, p(x, 0)=1$, using $N$ equally spaced cells with different fluxes, $t=2, L_{1}$ and $L_{\infty}$ errors of density $\rho$.

\begin{tabular}{|c|c|c|c|c|c|c|c|}
\hline$N$ & Flux & $L_{1}$ error & $L_{1}$ order & error ratio & $L_{\infty}$ error & $L_{\infty}$ order & Error ratio \\
\hline \multirow[t]{9}{*}{10} & $\mathrm{LF}$ & $5.74 \mathrm{E}-03$ & & 1.0000 & $8.12 \mathrm{E}-03$ & & 1.0000 \\
\hline & LLF & $5.38 \mathrm{E}-03$ & & 0.9373 & $7.75 \mathrm{E}-03$ & & 0.9544 \\
\hline & G & $5.38 \mathrm{E}-03$ & & 0.9373 & $7.75 \mathrm{E}-03$ & & 0.9544 \\
\hline & $\mathrm{EO}$ & $5.38 \mathrm{E}-03$ & & 0.9373 & $7.75 \mathrm{E}-03$ & & 0.9544 \\
\hline & HLL & $6.05 \mathrm{E}-03$ & & 1.0540 & 8.59E-03 & & 1.0579 \\
\hline & HLLC & $5.38 \mathrm{E}-03$ & & 0.9373 & $7.75 \mathrm{E}-03$ & & 0.9544 \\
\hline & FORCE & $6.55 \mathrm{E}-03$ & & 1.1411 & $8.96 \mathrm{E}-03$ & & 1.1034 \\
\hline & FLIC & $4.56 \mathrm{E}-03$ & & 0.7944 & 7.81E-03 & & 0.9618 \\
\hline & MUSTA & $6.86 \mathrm{E}-03$ & & 1.1951 & $9.27 \mathrm{E}-03$ & & 1.1416 \\
\hline \multirow[t]{9}{*}{20} & $\mathrm{LF}$ & $2.77 \mathrm{E}-04$ & 4.37 & 1.0000 & $4.41 \mathrm{E}-04$ & 4.20 & 1.0000 \\
\hline & LLF & $2.56 \mathrm{E}-04$ & 4.40 & 0.9242 & $4.12 \mathrm{E}-04$ & 4.23 & 0.9342 \\
\hline & G & $2.56 \mathrm{E}-04$ & 4.40 & 0.9242 & $4.12 \mathrm{E}-04$ & 4.23 & 0.9342 \\
\hline & $\mathrm{EO}$ & $2.56 \mathrm{E}-04$ & 4.40 & 0.9242 & $4.12 \mathrm{E}-04$ & 4.23 & 0.9342 \\
\hline & HLL & $2.94 \mathrm{E}-04$ & 4.36 & 1.0614 & $4.78 \mathrm{E}-04$ & 4.17 & 1.0839 \\
\hline & HLLC & $2.56 \mathrm{E}-04$ & 4.40 & 0.9242 & $4.12 \mathrm{E}-04$ & 4.23 & 0.9342 \\
\hline & FORCE & $3.21 \mathrm{E}-04$ & 4.35 & 1.1588 & $5.00 \mathrm{E}-04$ & 4.16 & 1.1338 \\
\hline & FLIC & $1.24 \mathrm{E}-04$ & 5.20 & 0.4477 & $2.53 \mathrm{E}-04$ & 4.95 & 0.5737 \\
\hline & MUSTA & $3.33 \mathrm{E}-04$ & 4.36 & 1.2022 & $5.16 \mathrm{E}-04$ & 4.17 & 1.1701 \\
\hline \multirow[t]{9}{*}{40} & $\mathrm{LF}$ & $8.54 \mathrm{E}-06$ & 5.02 & 1.0000 & $1.58 \mathrm{E}-05$ & 4.80 & 1.0000 \\
\hline & LLF & $7.86 \mathrm{E}-06$ & 5.02 & 0.9204 & $1.46 \mathrm{E}-05$ & 4.82 & 0.9241 \\
\hline & G & $7.86 \mathrm{E}-06$ & 5.02 & 0.9204 & $1.46 \mathrm{E}-05$ & 4.82 & 0.9241 \\
\hline & $\mathrm{EO}$ & 7.86E-06 & 5.02 & 0.9204 & $1.46 \mathrm{E}-05$ & 4.82 & 0.9241 \\
\hline & HLL & $9.14 \mathrm{E}-06$ & 5.01 & 1.0703 & $1.79 \mathrm{E}-05$ & 4.74 & 1.1329 \\
\hline & HLLC & 7.86E-06 & 5.02 & 0.9204 & $1.46 \mathrm{E}-05$ & 4.82 & 0.9241 \\
\hline & FORCE & $9.90 \mathrm{E}-06$ & 5.02 & 1.1593 & $1.81 \mathrm{E}-05$ & 4.79 & 1.1456 \\
\hline & FLIC & $3.30 \mathrm{E}-06$ & 5.23 & 0.3864 & $8.22 \mathrm{E}-06$ & 4.94 & 0.5203 \\
\hline & MUSTA & $1.02 \mathrm{E}-05$ & 5.03 & 1.1944 & $1.86 \mathrm{E}-05$ & 4.80 & 1.1772 \\
\hline \multirow[t]{9}{*}{80} & $\mathrm{LF}$ & $2.66 \mathrm{E}-07$ & 5.00 & 1.0000 & $4.96 \mathrm{E}-07$ & 4.99 & 1.0000 \\
\hline & LLF & $2.45 \mathrm{E}-07$ & 5.00 & 0.9211 & $4.56 \mathrm{E}-07$ & 5.00 & 0.9194 \\
\hline & G & $2.45 \mathrm{E}-07$ & 5.00 & 0.9211 & $4.56 \mathrm{E}-07$ & 5.00 & 0.9194 \\
\hline & $\mathrm{EO}$ & $2.45 \mathrm{E}-07$ & 5.00 & 0.9211 & $4.56 \mathrm{E}-07$ & 5.00 & 0.9194 \\
\hline & HLL & $2.85 \mathrm{E}-07$ & 5.00 & 1.0714 & $5.77 \mathrm{E}-07$ & 4.95 & 1.1633 \\
\hline & HLLC & $2.45 \mathrm{E}-07$ & 5.00 & 0.9211 & $4.56 \mathrm{E}-07$ & 5.00 & 0.9194 \\
\hline & FORCE & $3.07 \mathrm{E}-07$ & 5.01 & 1.1541 & $5.72 \mathrm{E}-07$ & 4.98 & 1.1532 \\
\hline & FLIC & 1.03E-07 & 5.00 & 0.3872 & $2.21 \mathrm{E}-07$ & 5.22 & 0.4456 \\
\hline & MUSTA & $3.12 \mathrm{E}-07$ & 5.02 & 1.1729 & $5.82 \mathrm{E}-07$ & 4.99 & 1.1734 \\
\hline \multirow[t]{9}{*}{160} & LF & $8.24 \mathrm{E}-09$ & 5.01 & 1.0000 & $1.47 \mathrm{E}-08$ & 5.08 & 1.0000 \\
\hline & LLF & 7.58E-09 & 5.01 & 0.9199 & $1.35 \mathrm{E}-08$ & 5.07 & 0.9184 \\
\hline & G & 7.58E-09 & 5.01 & 0.9199 & $1.35 \mathrm{E}-08$ & 5.07 & 0.9184 \\
\hline & $\mathrm{EO}$ & 7.58E-09 & 5.01 & 0.9199 & $1.35 \mathrm{E}-08$ & 5.07 & 0.9184 \\
\hline & HLL & 8.82E-09 & 5.01 & 1.0704 & $1.72 \mathrm{E}-08$ & 5.07 & 1.1701 \\
\hline & HLLC & $7.58 \mathrm{E}-09$ & 5.01 & 0.9199 & $1.35 \mathrm{E}-08$ & 5.07 & 0.9184 \\
\hline & FORCE & $9.47 \mathrm{E}-09$ & 5.02 & 1.1493 & $1.69 \mathrm{E}-08$ & 5.08 & 1.1497 \\
\hline & FLIC & 3.19E-09 & 5.02 & 0.3871 & 7.02E-09 & 4.97 & 0.4776 \\
\hline & MUSTA & $9.58 \mathrm{E}-09$ & 5.03 & 1.1626 & $1.71 \mathrm{E}-08$ & 5.09 & 1.1633 \\
\hline
\end{tabular}



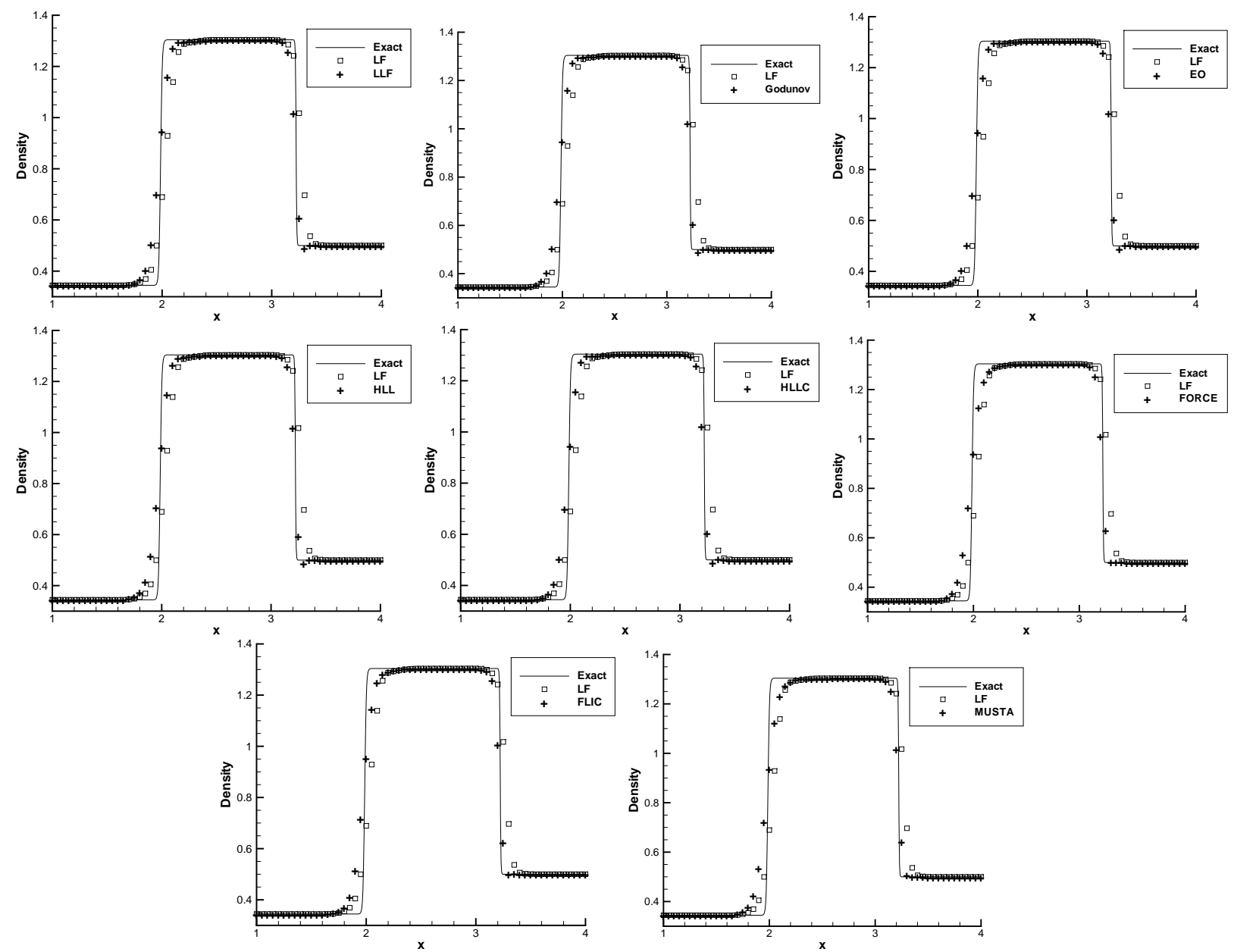

Figure 3.1: Solutions of Lax problem using WENO method and various fluxes considered: panels present comparison of numerical fluxes LLF, Godunov, EO, HLL, HLLC, FORCE, FLIC, MUSTA respectively with LF numerical flux and exact solution. In all cases 200 points were used. The solid line: exact solution; Plus: computed solution by different fluxes; Square: computed solution by LF flux. 


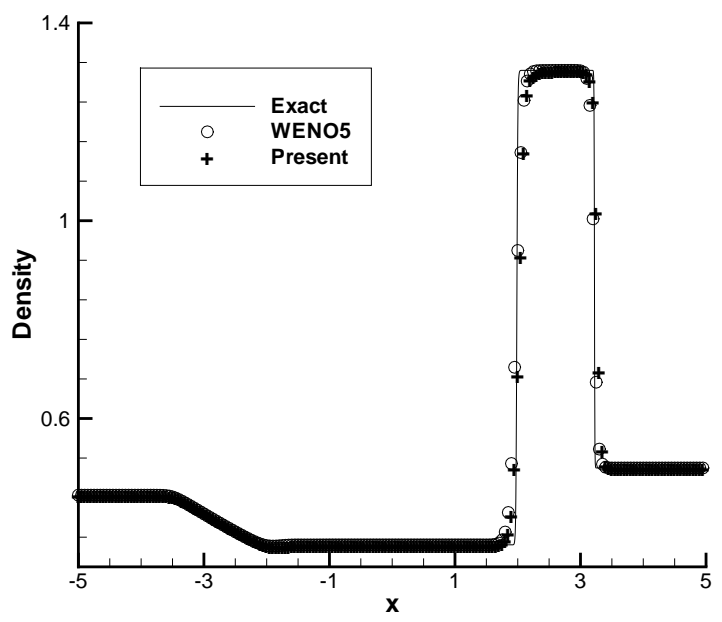

Figure 3.2: The Euler equations Lax problem. Solid line: exact solution; circle: computed solution of WENO5 with LF flux, plus: computed solution of our scheme.

is not known for this problem. As for this test, a zoomed result at the region $[0.5,2.5]$ is given, which contains the complicated wave pattern in the smooth part of the solution.

From Fig.3.3, we see that all (other) schemes describe the complicated wave pattern better than the LF scheme (such as the resolution). In Fig. 3.4, we show the numerical results by standard WENO-LF discretization scheme and our scheme, we can see that the results by method with alternative WENO-LF has better performance than the standard WENO-LF scheme.

Example 3.4. Blast-wave problem. Here test consists of the interaction of the blast wave of Euler equation in the domain $0 \leq x \leq 1$ with the initial condition

$$
(\rho, v, p)= \begin{cases}(1,0,1000) & \text { for } 0 \leq x<0.1 \\ (1,0,0.01) & \text { for } 0.1 \leq x<0.9 \\ (1,0,100) & \text { for } x \geq 0.9\end{cases}
$$

A reflecting boundary condition is applied to both ends. We solve this problem numerically on a domain discretised by 400 equally grid points until the final time $t=0.038$. The densities are plotted in Fig. 3.5 against the reference solution (the solid line), which is computed by the WENO scheme with 2000 grid points, and against the solution computed by the WENO-LF scheme on the same mesh, zoomed at the region $[0.53,0.88]$ which contains the contact discontinuities and shocks in the solution. Although the correction scheme (essentially two point) have better matching than before (before correction) against the exact resolution, we can clearly see that the WENO-LF scheme has the worst resolution, and that the HLLC, Godunov and EO fluxes based on our scheme have higher resolution than other fluxes considered. In Fig. 3.6, we show the numerical results by standard WENO-LF discretization scheme and our scheme, we can see that the results by method with alternative 

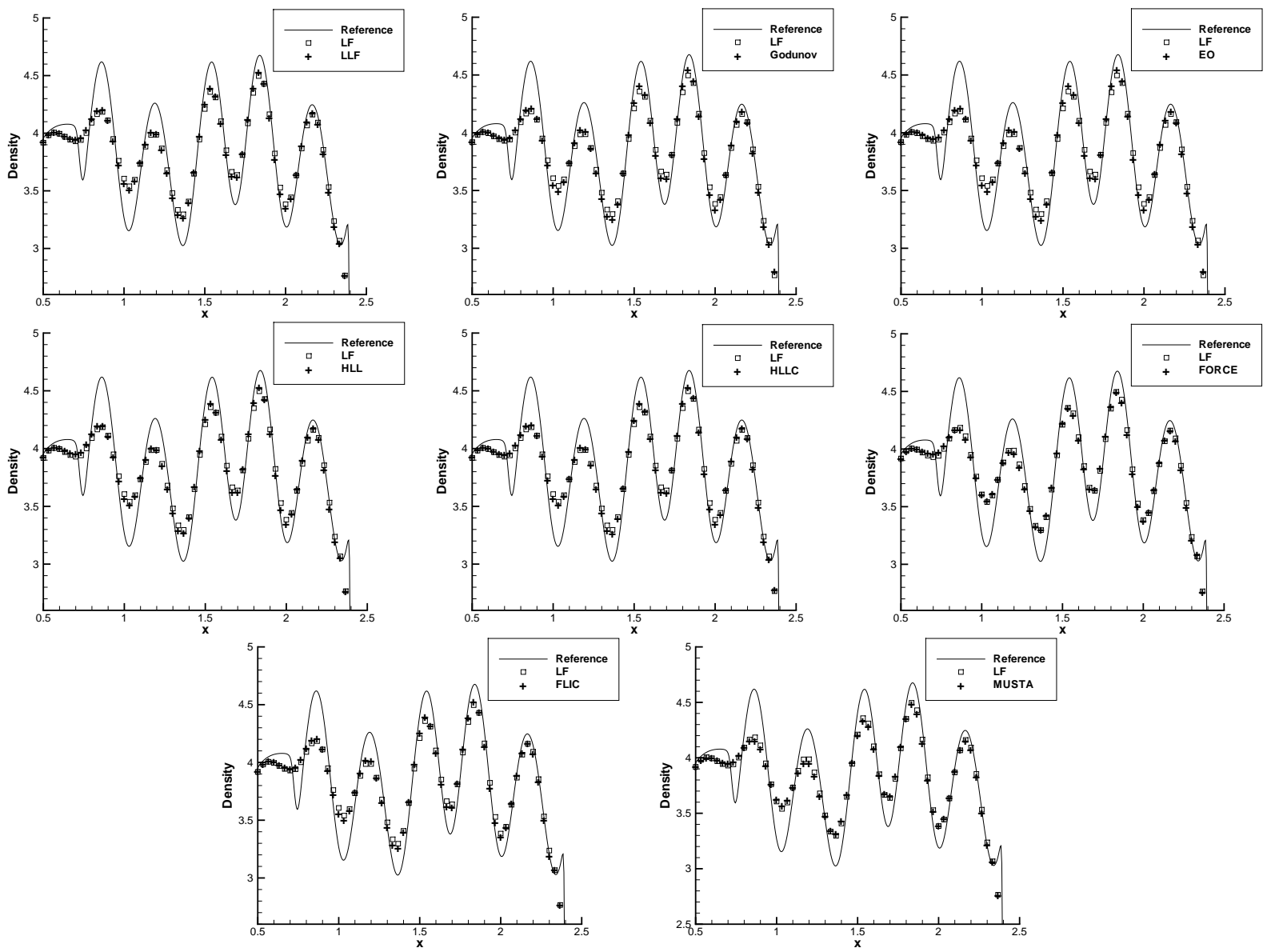

Figure 3.3: Density profile of the Shu-Osher problem using WENO method and various fluxes considered: panels show comparison of LLF, Godunov, EO, HLL, HLLC, FORCE, FLIC, MUSTA respectively with LF numerical flux and the "exact" reference solution. In all cases 300 points were used. The solid line: the "exact" reference solution; Plus: the results computed by different fluxes; Square: the results computed by LF flux. 


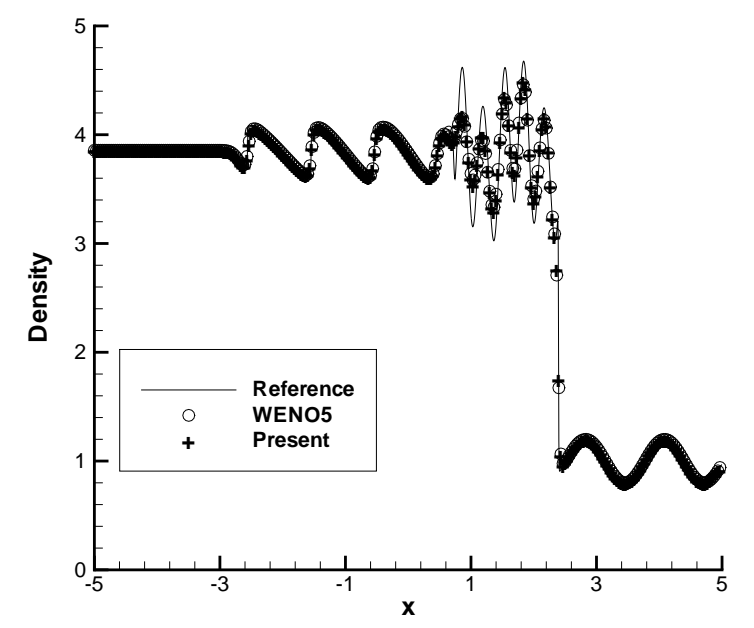

Figure 3.4: The Euler equations Shu-Osher problem. Solid line: the "exact" reference solution; circle: the results computed by WENO5 with LF flux, plus: our scheme.

Table 3.3: CPU time (in hours) for the WENO methods for the double Mach reflection problem. Total $\mathrm{CPU}$ time for the different grids is recorded.

\begin{tabular}{|c|c|c|c|c|}
\hline flux & $240 \times 80$ & $480 \times 160$ & $960 \times 240$ & $1920 \times 480$ \\
\hline LF & 0.1045 & 0.6182 & 4.8832 & 39.3199 \\
HLL & 0.1047 & 0.6016 & 4.9928 & 39.1599 \\
HLLC & 0.1099 & 0.6286 & 5.1345 & 41.4207 \\
\hline
\end{tabular}

WENO-LF has better performance than the standard WENO-LF scheme.

Example 3.5. Double Mach reflection. As an example of two dimensional problem we study double Mach reflection, which was extensively studied by Woodward and Colella in [22] and later by many others. We use the same setup as in [22], namely, the reflection of a Mach 10 planar shock by a wedge having a half-angle of $30^{\circ}$. The problem was solved on the $[0,4] \times[0,1]$ domain. Initially a right-moving Mach 10 shock is located at $x=\frac{1}{6}, y=0$ and forms a $60^{\circ}$ angle with $\mathrm{x}$-axis. The exact post-shock condition is composed on the lower boundary starting at $x=0$ to $x=\frac{1}{6}$ and reflecting boundary condition is used on the rest of the boundaries. The flow values are set to describe the exact motion of a Mach 10 shock at the top boundary. We compute the solution up to $t=0.2$.

In this example we have enhance three fluxes, namely LF, HLL and HLLC flux, which performed best among all other fluxes in one dimensional case. The results of the CPU times for various system sizes are given in table 3.3 with different meshes. Note that all three numerical fluxes take similar CPU time. The results at final time $t=0.2$ are shown in Fig. 3.7 with $960 \times 240$.

Example 3.6. Forward facing step problem [22]. The setup of the problem is as follows. The wind tunnel is 1 length unit wide and 3 length units long. The step is 0.2 length units 

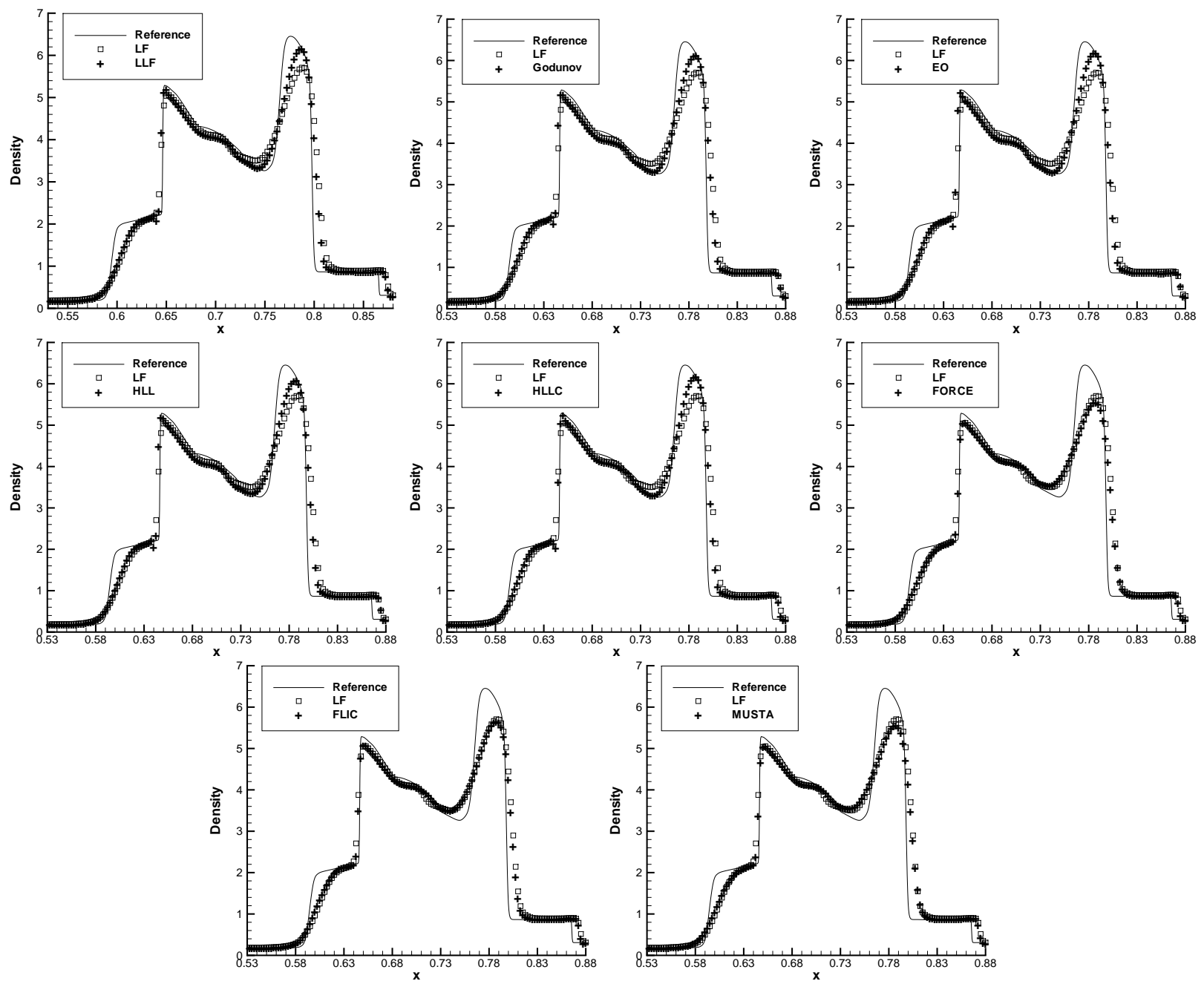

Figure 3.5: Density profile of the Blast-wave problem using WENO method and various fluxes considered: panels show comparison of LLF, Godunov, EO, HLL, HLLC, FORCE, FLIC, MUSTA respectively with LF numerical flux and the "exact" reference solution. In all cases 400 points were used. The solid line: the "exact" reference solution; Plus: the results computed by different fluxes; Square: the results computed by LF flux. 


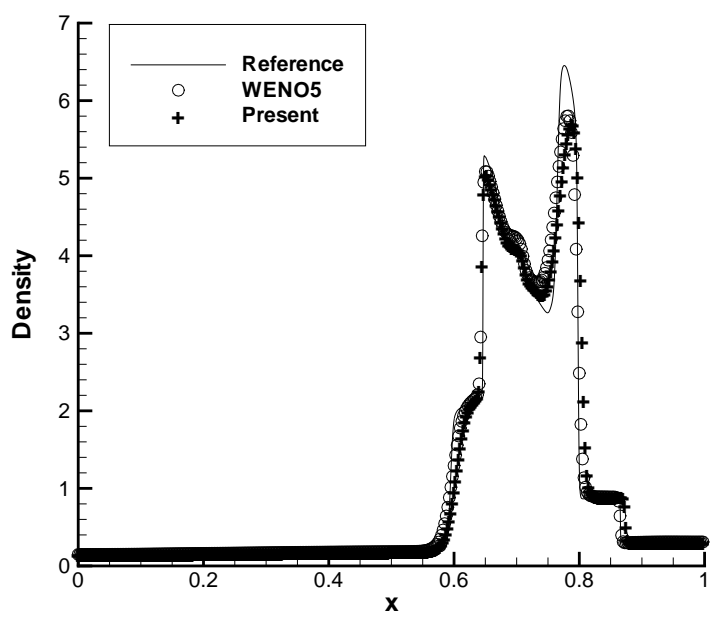

Figure 3.6: The Euler equations Blast-wave problem. Solid line: the "exact" reference solution; circle: the results computed by WENO5 with LF flux, plus: our scheme.

high and is located 0.6 length units from the left-hand end of the tunnel. The problem is initialized by a right-going Mach 3 flow. Reflective boundary conditions are applied along the wall of the tunnel and in and out flow boundary conditions are applied at the entrance (left-hand end) and the exit (right-hand end). The corner of the step is a singular point and we treat it the same way as in [22], which is based on the assumption of a nearly steady flow in the region near the corner.

The results are shown at $t=4.0$ by three different numerical fluxes, see Fig. 3.8. All the figures are showing 30 equally spaced density contours from 1.5 to 22.7 . It seems that HLLC scheme perform similarly well for this test case. And the HLLC scheme have the best resolutions among all schemes.

\section{Concluding remarks}

In this paper, we used an alternative numerical fluxes, based on WENO scheme, and compared the performance of eight different two point or essentially two point numerical fluxes. The method of the alternative numerical flux is simple and efficient. Numerical results show pros and cons of different fluxes for the different problems considered. Extensive one and two dimensional simulations on the hyperbolic systems of Euler equations indicate that the LF flux cost the least CPU time among all, but the numerical errors and resolution of solutions on the discontinuities are also the worst among all. The Godunov or EO fluxes seem to cost significantly more CPU time than the LF flux. The HLL and HLLC fluxes might be good choices as fluxes for our method when considered all factors, such as the cost of CPU time, numerical errors and resolution of discontinuities in the solution are considered. 

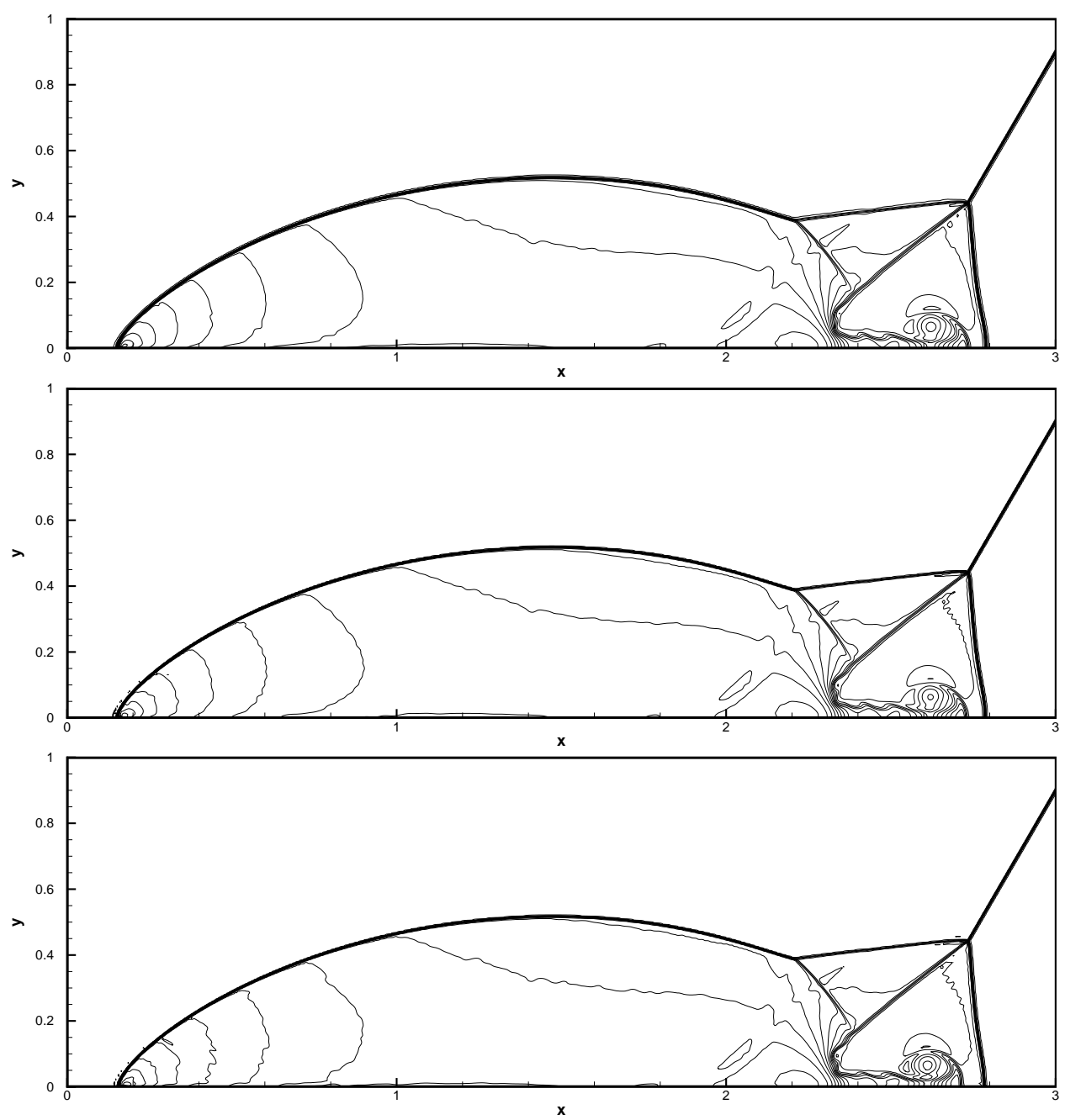

Figure 3.7: Double Mach reflection problem. Density contours for LF, HLL and HLLC numerical fluxes are shown in panels respectively. 30 density contours are equally spaced between 1.5 to 22.7 on a system size $960 \times 240$. WENO-LF (top), WENO-HLL (middle) and WENO-HLLC (bottom). 

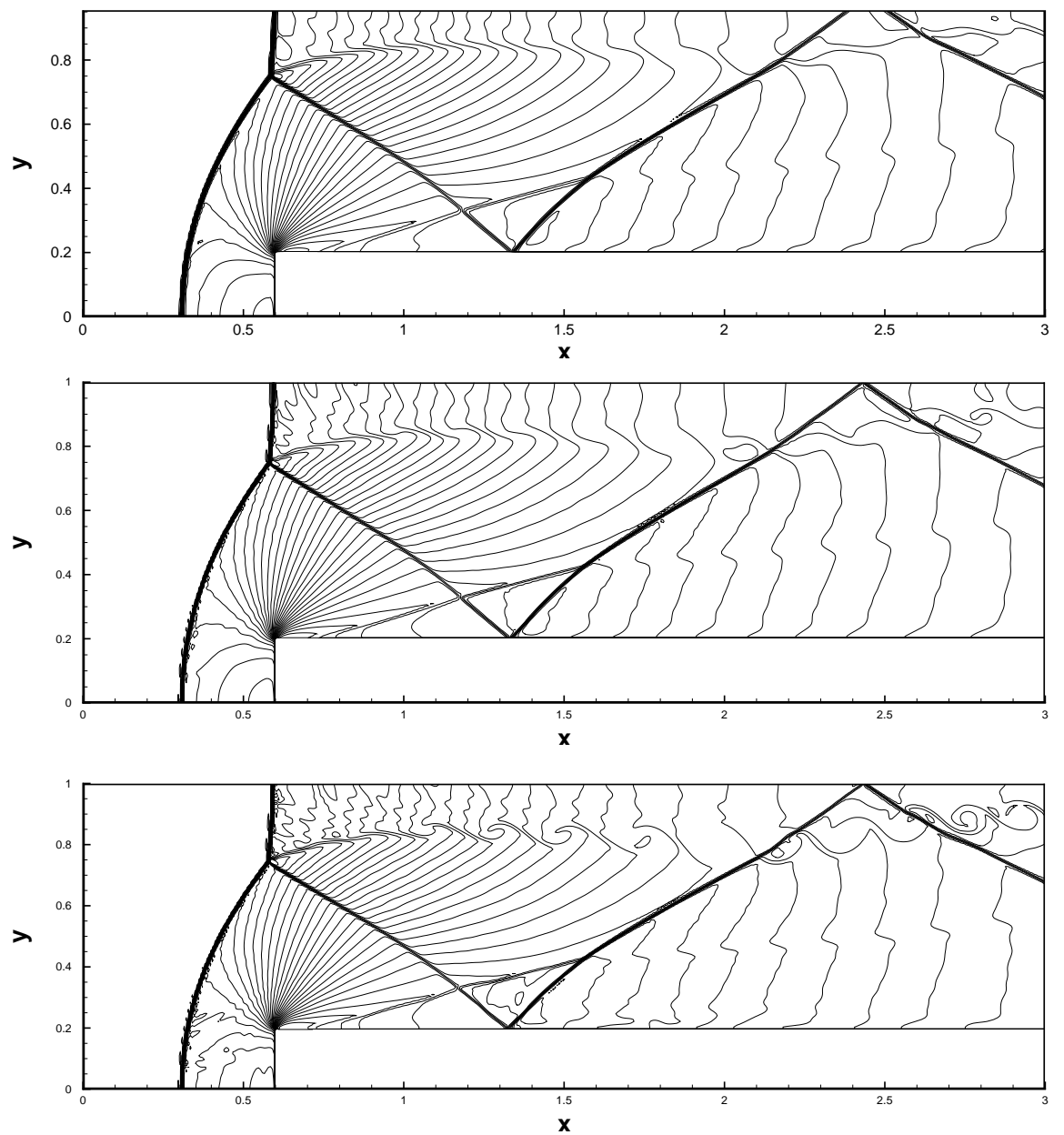

Figure 3.8: Forward step problem problem. Density contours for LF, HLL and HLLC numerical fluxes are shown in panels respectively. 30 density contours are equally spaced between 0.32 to 6.15 on a system size $480 \times 160$. WENO-LF (top), WENO-HLL (middle) and WENO-HLLC (bottom). 


\section{References}

[1] D. Balsara and C.-W. Shu, Monotonicity preserving weighted essentially non-oscillatory schemes with increasingly high order of accuracy, J. Comput. Phys., 160 (2000), 405-452.

[2] P. Batten, M.A. Leschziner and U.C. Goldberg, Average-state Jacobians and implicit methods for compressible viscous and turbulent flows, J. Comput. Phys., 137 (1997), 38-78.

[3] B. Engquist and S. Osher, One sided difference approximation for nonlinear conservation laws, Math. Comp., 36 (1981), 321-351.

[4] S.K. Godunov, Finite difference methods for the computation of discontinuous solutions of the equations of fluid dynamics, Math. Sbornik, 47 (1959), 271-306.

[5] A. Harten, P.D. Lax and B. van Leer, On upstream differencing and Godunov-type schemes for hyperbolic conservation laws, SIAM Rev., 25 (1983), 35-61.

[6] G. Jiang, C.-W. Shu, Efficient implementation of weighted ENO schemes, J. Comput. Phys., 126 (1996), 202-228.

[7] Y. Jiang, C.-W. Shu, and M.P. Zhang, An alternative formulation of finite difference weighted ENO schemes with Lax-Wendroff time discretization for conservation laws, SIAM J. Sci. Comput., 35 (2013), 137-160.

[8] X.D. Liu, S. Osher, and T. Chan, Weighted essentially non-oscillatory schemes, J. Comput. Phys., 115 (1994), 200-212.

[9] H. Liu and J. Qiu, Finite Difference Hermite WENO Schemes for Conservation Laws, II: an Alternative Approach, J. Sci. Comput., 66 (2016), 598-624.

[10] S. Osher and F. Solomon, Upwind difference schemes for hyperbolic conservation laws, Math. Comp., 38 (1982),339-374.

[11] J. Qiu, A Numerical comparison of the Lax-Wendroff discontinuous Galerkin Method Based on Different Numerical Fluxes, J. Sci. Comput., 30 (2007), 345-367.

[12] J. Qiu, B.C. Khoo, and C.-W. Shu, A Numerical study for the performance of the RungeKutta discontinuous Galerkin method based on different numerical fluxes. J. Comput. Phys., 212 (2006), 540-565. 
[13] J. Qiu, C.-W. Shu, Finite difference WENO schemes with Lax-Wendroff type time discretizations, SIAM J. Sci. Comput., 24 (2003), 2185-2198.

[14] C.-W. Shu, Essentially non-oscillatory and weighted essentially non-oscillatory schemes for hyperbolic conservation laws, NASA/CR-97-206253, ICASE Report NO.97-65.

[15] C.-W. Shu, High order weighted essentially nonoscillatory schemes for convection dominated problems, SIAM Rev., 51 (2009), 82-126.

[16] C.-W. Shu, and S. Osher, Efficient implenmentiation of essentially non-oscillatory shock-capturing schemes, J. Comput. Phys., 77 (1988), 439-471.

[17] C.-W. Shu, and S. Osher, Efficient implenmentiation of essentially non-oscillatory shock-capturing schemes, II, J. Comput. Phys., 83 (1989), 32-78.

[18] V.A. Titarev and E.F. Toro, Finite-volume WENO schemes for three-dimensional conservation laws, J. Comput. Phys., 201 (2004), 238-260.

[19] E.F. Toro, Riemann solvers and numerical methods for fluid dynamics, a practical introduction, Springer, Berlin, 1997.

[20] E.F. Toro, Multi-stage predictor-corrector fluxes for hyperbolic equations, Preprint NI03037-NPA, Isaac Newton Institute for Mathematical Sciences, University of Cambridge, UK.

[21] E.F. Toro, M. Spruce, and W. Speares, Restoration of the contact surface in the HartenLax van Leer Riemann solver, J. Shock Waves, 4 (1994), 25-34.

[22] P. Woodward and P. Colella, The numerical simulation of two-dimensional fluid flow with strong shocks, J. Comput. Phys., 54 (1984), 115-173. 